\title{
Téoros
}

Revue de recherche en tourisme

\section{Les voyages-motivation : un phénomène en croissance aux} États-Unis

\section{Pierre Bellerose}

Volume 9, numéro 2, juillet 1990

Le marché américain

URI : https://id.erudit.org/iderudit/1080027ar

DOI : https://doi.org/10.7202/1080027ar

Aller au sommaire du numéro

Éditeur(s)

Université du Québec à Montréal

ISSN

0712-8657 (imprimé)

1923-2705 (numérique)

Découvrir la revue

Citer cet article

Bellerose, P. (1990). Les voyages-motivation : un phénomène en croissance aux États-Unis. Téoros, 9(2), 16-18. https://doi.org/10.7202/1080027ar d'utilisation que vous pouvez consulter en ligne.

https://apropos.erudit.org/fr/usagers/politique-dutilisation/ 


\section{Les voyages-motivation: un phénomène en croissance aux Etats-Unis}

La notion de rendement a toujours été une idee-force ausein desentreprises. Lamesure du rendement fait appel à des critères qui ont beaucoup changé au fil de l'évolution des philosophies et des modes de gestion.

Des firmes spécialisées - le Groupe Hay, William M. Mercer, Sobeco, etc. - ont développé une expertise pour aider les entreprises à choisir les mécanismes incitatifs les plus appropriés à chacune d'entre elles.

Toute entreprise est faite pour faire du profit. Mais de plus en plusd'entrepreneurs et de gestionnaires comprennent que la différence dans la compétition se feramoins par la technologie que par les ressources humaines (compétence, créativité, professionnalisme) et leurs motivations.

On a largement remplacé la technique de la carotte et du batton par des formes de participation aux bénéfices (bonus, partage des profits, achat d'actions et autres).

Il n'y a sûrement pas de formule-miracle mais une des plus anciennes formes de stimulation de certaines catégories d'employés est le voyage-motivation. II s'agit d'un phénomène assez mal connu par nombre d'intervenants touristiques. II représente pourtant un segment important et lucratif: les agences de voyage spécialiscées dans les comptes commerciaux, les transporteurs et certains agents opérateurs à destination-entre autres Gerry Lou, SAT, Réceptour au Québec - l'ont compris depuis plusieurs années. Force est d'admettre cependant qu'il s'agit d'un marché très fragmenté et, par le fait même, plus difficile à mesurer.

\section{Historique}

Le concept de la motivation a été introduit aux État-Unis durant la crise économique des années ' 30 . Comme les ventes étaient a la baisse et qu'il fallait motiver les employés, deux innovateurs, E.F. MacDonald de Fayton (Ohio) et Maritz (N.Y.), insèrent un système de cadeaux-récom-

Pierre Bellerose est directeur de la recherche et du développement a l'Office des congras et du touriame du Grand Montréal (OCTGM). penses liess aux résultats des ventes. Le voyage n'aété utilisé qu'en 1935 par General Electric qui organisa une croisière aux Bermudes pour ses meilleurs agents commerciaux. Cependant, ce n'est qu'à partir des années ' 50 qu'on a observé le début de l'expansion du concept. À cette époque, les voyages-motivation étaient un phénomene purement et uniquement américain. On ne retrouvait pas $1^{t}$ equivalent ailleurs et les Américains ne voyageaient pratiquement qu'aux État-Unis. Ce n'est que dans les années '60, avec l'amélioration des moyens de transport, que nos voisins du Sud ont commencé à sortir de leurs pays et, par ricochet, ont facilité $1^{7}$ éclosion de petites industries de voyages-motivation au Canada, au Québec et en Europe.

Historiquement, depuis pres de trente ans, la presque totalité de la clientele qui vient au Québecest américaine, et cela pourdeux. raisons:

- c'est le marché le plus important et le plus payant;

- le Québec est different (européen), donc exotique, et se prête bien aux voyages-motivation des Américains.

Aux Êtat-Unis, entre 1964 et 1974 , le chiffre d'affaires de ce nouveau marché aurait décuplé selon certaines estimations ${ }^{(1)}$, passant de $\$ 50$ à $\$ 500$ millions (U.S.). En 1980, le milliard a été dépassé et selon Premium Incentive Business, le chiffre d'affaires s'eleverait a $\$ 4,6$ milliards en 1988.

\section{Tendances du marché américain}

Aux Etat-Unis; la Society of Incentive Travel Executives (SITE) produit les informations et les statistiques les plus valables sur ce sujet grâce à une enquête annuelle auprès de ses membres. SITE aété fondée en 1973 pour répondre aux besoins a la fois des responsables de voyages-motivation des differrentes entreprises et à ceux des intermédiaires qui offrent différents produits à ces entreprises.

Des 53 millions d'Américains qui ont pris l'avion sur une ligne commerciale en 1987 , plus de 3 millions (ou $6 \%$ ) de ce total avaient comme motif premier le voyage- motivation ${ }^{(2)}$. Approximativement $61 \%$ des Américains ont effectué leur voyagemotivation aux État-Unis en $1988,14 \%$ dans les Caraibes et $25 \%$ ailleurs dans le monde (dont le Canada).

Pour les voyages-motivation, le terme client fait référence à des entreprises plutôt qu'à des personnes. En effet, les ventes se font directement auprès d'entreprises qui désirent récompenser leurs employés.

Au plan géographique, les sièges sociaux des corporations utilisant les voyagesmotivation se situent dans les grandes regions suivantes: Nouvelle-Angleterre et Atlantique Centre $(31 \%)$, Centre Nord-Est et Centre Nord-Ouest $(35,5 \%)$, CentreSudOuest $(15,5 \%)$, Centre Sud-Est et Atlantique Sud $(8 \%)$, Montagnes et Pacifique $(10 \%)$

On notera que le Québec est à proximité du plus grand bassin de sieges sociaux au monde (les deux tiers sont à sa portée) en ce qui concerne les voyages-motivation, ce qui devient un avantage s'il arrive à se differencier de là concurrence.

Selon SITE, les voyages-motivation sont utilisés par virtuellement tous les secteurs d'activités mais une douzaine de secteurs majeurs composent les deux tiers du chiffre d'affaires total. Ce sont des industries qui possèdent un système marketing sophistiqué et emploient plusieurs niveaux de vendeurs, distributeurs et négociants(s). L'employe moyen travaille seul et le voyage-motivation devient un excellent véhicule de rencontre entre les employés et les cadres.

Le tableau 1 montre que les dix premières industries en termes devoyages-motivation génèrent près 3,1 milliards de dollars en 1988 .

En 1988, le coût moyen d'un voyage-motivation payé par une entreprise américaine s'élevait à 1611 \$ (U.S.) par personne. Ilest à signalerque $90 \%$ des programmes prévoit que le "boni-voyage" inclut le conjoint ou un invite, ce qui augmente sensiblement l'impact économique du phenomene. Le tableau 2 permet d'évaluer cet impact.

Seulement $32 \%$ des voyages couttent plus de 1800 \$ et ce sont sans doute ceux qui sont effectuésendehors de $1^{3}$ Amérique duNord. 
Le marché potentiel pour le Québec, en considérant les coûts, serait de près de $40 \%$ du total (coût de $500 \$$ a $1500 \$$ ).

La durée du séjour moyen était de 5 nuitsen 1989.

\section{Caractéristiques des voyages}

Il existe deux grands types de voyagesmotivation, soit celui pour les vendeurs ("salesforce trips") et celui pour les négociants ("dealer trips"). Les deux types de voyages connaissent des hausses régulières depuis 1982 bien que la proportion des voyages augmente plus rapidement dans le cas des vendeurs que des négociants.

Une partie du voyage-motivation est consacrée à des réunions de travail. Bien que l'ensemble du voyage en soit un d'agrément, on consacre en moyenne près de dix heures (en 1987) en réunions professionnelles. Cependant, ce phénomene n'est pas uniforme pour toutes les entreprises comme le montre le tableau 3.

Ces données seraient en hausse depuis quelques années. Une des raisons qui expliquent cette augmentation est d'ordre fiscal. Eneffet, si le voyage sert uniquement à des fins d'agrément, il est considéré, au plan de la fiscalité américaine, comme un bonus pour l'employé et constitue donc, par conséquent, un revenu imposable pour ce dernier. Le fait d'inclure une session de travail permet alors à l'entreprise de considérer le voyage comme étant une dépense d'entreprise et n'influence en rien le revenu imposable des employes. Des conférenciers, plus volontiers appelés motivateurs, sont embauchés en vue de gonfler le moral des forces de ventes.

La moitié (49\%) des voyages-motivation a lieu entre les mois de janvier et avril inclusivement. Ceci s'explique en partie par le fait que les industries ont une "basse saison" quant à leurs activités et qu'il est plus facile de libérer leurs employés pour une semaine (voir tableau 4). Cependant, en ce qui concerne les voyages-motivation des Américains au Québec, la "haute saison" est située en été.

\section{Processus décisionnel}

L'augmentation du volume des ventes est de loin 1'objectif le plus important pour les entreprises qui offrent des voyagesmotivation à leurs employés comme le montre le tableau 5. D'autres objectifs sont également poursuivis. Il est à noter que l'objectif "augmenter l'intérêt et la motivation" n'est pas l'objectif principal poursuivi par les entreprises etque laplupart des objectifs sont liés à la vente.

TABLEAU 1

Principaux utilisateurs des voyages-motivation, 1988

Industries

Recettes (dollars U.S.)

1- Alimentation 651 millions

2- Assurances 504 millions

3- Automobiles, bateaux, avions

4- Accessoires automobiles 404 millions

5- $\quad$ Produits pharmaceutiques 362 millions

6- $\quad$ Biens non durables (grossistes) 165 millions

7- Magazines 154 millions

8- Bien durables (grossistes) 150 millions

9- Édition 141 millions

10- Quincaillerie 127 millions

Source: Premium/Incentive Business, juillet 1989.

N.B.: Une des sources les plus fiables puisqu ${ }^{4}$ il $\mathrm{s}^{7}$ agit $\mathrm{d}^{7} u n$ échantillon de plus de 1000 entreprises américaines.

Toujours selon Incentive Magazine, le choix d'une destination repose sur plusieurs facteurs, dont les plus importants sont les suivants:

1- Le budget, facteur crucial.

2- La periode de $1^{2}$ année la plus propice pour ces voyages, chaque industrie ayant une "basse saison" permettant aux employés d'être absents du travail.

3- Les affaires ou l'agrément, selon l'emphase qu'on desire donner aux voyages;

4- Les caracteristiques des gagnants sont prises en considération, compte tenu de leurs expériences personnelles de voyage.

5- L'accessibilité afin que le groupe puisse se rendre à destination en tout confort.

6- L'infrastructure de services.

7- Les choses à faire.

8- L'expertise des fournisseurs dans le domaine des voyages-motivation.

9- Les équipements sportifs et récréatifs qui ajoutent à l'attrait d'une destination.

La décision du choix de la destination est prise par les dirigeants des entreprises. Les responsables qui exercent la plus grande influence sont le président, le vice-président exécutif et le directeur général dans $64 \%$ des cas, le directeur des ventes et le directeur du marketing dans une proportion de $28 \%$ et les autres responsables pour $8 \%$.

\section{Mise en marché}

La mise en marché de tels voyages sur le marché américain ne se fait pas aisément: elle demande une bonne expertise, des contacts sérieux, une bonne réputation et un programme innovateur afin d'impressionner les groupes.

Les acteurs sont nombreux lorsqu'il s'agit de vendre un voyage-motivation. Il y a d'abord $1^{*}$ entreprise (acheteur) qui désire offrir un voyage pour ses employés ou clients, son mandataire ("Incentive house") qui a pour tâche d'élaborer un programme d'activités et une liste de destinations possibles, les fournisseurs de services (transporteurs, hôteliers, etc.) et les représentants de la destination (agents réceptifs, bureaux de tourisme) qui ont pour but de vendre les attraits de leur destination.

Pour un vendeur, les possibilités de prendre contact avec des dirigeants d'entreprises ou avec des spécialistes des maisons de motivation sont nombreuses. Une participation aux activités de LIMRA (Life Insurance Marketing \& Research Association), ICPA (Insurance Conference Planners Association) et ITME (Incentive Travel \& Marketing Executive Show) procure ce genre de possibilités.

Les toumées de familiarisation sont un autre moyen de faire valoir un produit. Ces voyages se planifient longtemps à l'avance (de 12 a 24 mois avant le voyage).

Il existe près d'une centaine de firmes spécialisées en voyages-motivation aux Etat-Unis (Incentive Travel Houses). Les deux plus importantes firmes sont Carlson Marketing Group et Maritzqui ont chacune un chiffre d'affaires de plus de $\$ 200$ millions lié uniquement aux voyages-motivation.

\section{Situation du Québec (et du Canada)}

Le Québec contrổlerait environ $1,3 \%$ du marché américain soit l'équivalent de $\$ 50$ millions. Sur l'échiquier canadien, le 
Québec se tire bien d'affaires puisqu'il possède environ $50 \%$ du marché $\hat{e}^{(4)}$ (le Canada attire $2,6 \%$ du total des voyagesmotivation américains). Le Québec a un seul concurrent important au Canada (Colombie-Britannique). Signalons que l'Ontario est peu présente sur ce marché puisqu'elle ne peut pas offrir un produit "différent" (comme le Québec avec son cachet européenetlaColombie-Britannique avec les Rocheuses).

Comme une des caractéristiques duvoyagemotivation est $1^{\prime}$ exotisme et la différence, on attire au Québec une clientèle essentiellement américaine. Cependant, on peut penser qu'il y aurait un potentiel appréciable au sein des petites et moyennes entreprises canadiennes et québécoises.

Nous pensons qu'on sous-estime grandement l'importance des voyagesmotivation au Québec par des entreprises québécoises et canadiennes. Certains interlocuteurs ont souligné qu'un grand nombre d'entreprises de taille modeste (plus de 20 employés) retiennent la formule du voyage-motivation parce qu'elle est facile d'application. Dans ce contexte, il est vraisemblable que plusieurs centres situés en milieu de villégiature accueillent non seulement des réunions d'affaires mais des groupes d'employés qui bénéficient d'une prime aurendement parle biaisd'unvoyage, frais du conjoint compris.

De plus, le Québec a un avantage sur ses autres concurrents canadiens, soit la bipolarisation Montréal-Québec qui permet la création de forfaits d'une durée de six nuits (quatre à Montréal et deux à Québec), formule idéale pour ce type de produit.

\section{Conclusion}

Un nombre grandissant d'entreprises, de plus en plus préoccupées par l'amélioration de la productivité, sont à l'heure de la planification organisationnelle et de la gestion du rendement.

Dans ce contexte, les programmes d"intéressement des vendeurs et autres employés se raffinent. Les divers régimes de participation (immédiate ou différée) aux benefices se multiplient. On cherche à reconnaître la performance et à développer un esprit de partenariat entre les employés et l'employeur.

Il va quasi de soi - gratification immédiate aidant - que le voyage-motivation sera toujours une formule populaire. Et s'il est évident que le Québec n'a pas nécessairement tousles charmes de certaines destinations exotiques ou prestigieuses, il possède plusieurs atouts et doit mener de

TABLEAU 2

Coût des voyages-motivation par personne en 1988

$\begin{array}{lr}\text { Moins de } 500 \$(1) & 6 \% \\ 500 \$-999 \$ & 17 \% \\ 1000 \$-1199 \$ & 16 \% \\ 1200 \$-1399 \$ & 12 \% \\ 1400 \$-1799 \$ & 17 \% \\ 1800 \$-2399 \$ & 16 \% \\ \text { Plus de } 2400 \$ & 16 \%\end{array}$

Source:Incentive magazine, 1989.

TABLEAU 3

Réunions de travail durant les voyages-motivation des entreprises américaines (1987)

$\begin{array}{ll}\text { Souvent } & 32 \% \\ \text { Quelques fois } & 39 \% \\ \text { Rarement } & 12 \% \\ \text { Jamais } & 17 \%\end{array}$

Source: Successful Meetings, février 1988, p. 65.

TABLEAU 4

Calendrier des voyages, 1987

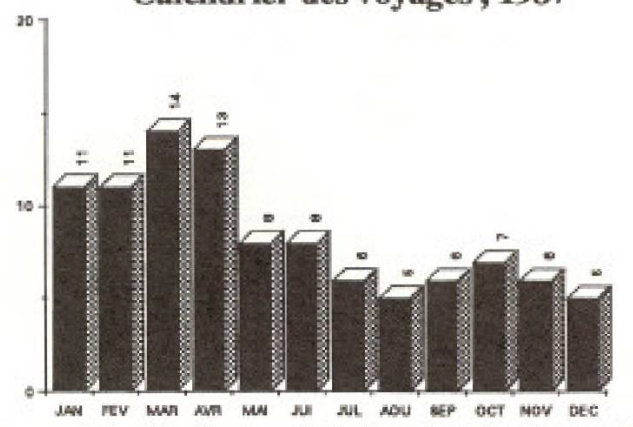

Source: Gouvernement du Québec, ministère du Tourisme, Profil de marché États-Unis, 1989 .

TABLEAU 5

Objectifs poursuivis par les woyages-motivation

$\begin{array}{lll}\text { 1- } & \text { Augmenter le volume des ventes } & 71 \% \\ 2- & \text { Augmenter l'intérêt et la motivation } & 47 \% \\ \text { 3- } & \text { Ouvrir de nouveaux comptes } & 37 \% \\ 4- & \text { Introduire de nouveaux produits } & 26 \% \\ 5- & \text { Mousser la vente d'une nouvelle gamme de produits } & 24 \% \\ \text { 6- } & \text { Concurrencer la promotion des compétiteurs } & 22 \% \\ 7- & \text { Se préparer pour une nouvelle saison de ventes } & 10 \%\end{array}$

Source: Incentive Magazine Survey, 1987.

façon continue son travail de connaissance et de sollicitation des clients. $f$

\section{NOTES}

(1) Voir Meetlings and Conventions, juin 1979, p= 52.
$71 \%$

$47 \%$

$37 \%$

$26 \%$

$22 \%$

$10 \%$
(2) Sueceseful Moetings, tóvrior $198 e_{i}$ p. 52.

(3) BAEDEMEIR, Judi, incentive Travel in the USA. Travel and Tourism Analyst, septembre 1986, p. 27.

(4) Selon lea diffórents apécialistes de ce marehé. 